\title{
Modified Takazawa anatomical classification of renal pelvicalyceal system based on three-dimensional virtual reconstruction models
}

\author{
Weijie Zhu ${ }^{1 \#}$, Mengmeng Zheng ${ }^{2 \#}$, Shengwei Xiong ${ }^{1 \#}$, Guanpeng Han ${ }^{1}$, Chang Meng ${ }^{1}$, Zhihua Li $^{1}$, \\ Lei Zhang ${ }^{1}$, Gengyan Xiong ${ }^{1}$, Hua Guan ${ }^{1}$, Yanbo Huang ${ }^{1}$, Hongjian Zhu ${ }^{3}$, Xuesong Li $^{1}$, Gang Wang ${ }^{1}$, \\ Liqun Zhou' ${ }^{1}$
}

${ }^{1}$ Department of Urology, Peking University First Hospital, Institute of Urology, Peking University, National Urological Cancer Centre, Beijing, China; ${ }^{2}$ Department of Urology, Beijing Friendship Hospital, Capital Medical University, Beijing, China; ${ }^{3}$ Department of Urology, Beijing Jiangong Hospital, Beijing, China

Contributions: (I) Conception and design: L Zhou, G Wang; (II) Administrative support: X Li; (III) Provision of study materials or patients: W Zhu, Z Li; (IV) Collection and assembly of data: W Zhu, M Zheng, S Xiong; (V) Data analysis and interpretation: S Xiong, H Zhu, G Xiong; (VI) Manuscript writing: All authors; (VII) Final approval of manuscript: All authors.

\#These authors contributed equally to this work.

Correspondence to: Xuesong Li; Gang Wang. Department of Urology, Peking University First Hospital, Beijing, China; Institute of Urology, Peking University, National Urological Cancer Center, No. 8 Xishiku St, Xicheng District, Beijing 100034, China. Email: pineneedle@sina.com; drwanggang@163.com.

Background: Previous classification of renal pelvicalyceal anatomical structure may be difficult to intuitively understand and unpractical for endourological surgery. We aim to put forward a modified Takazawa anatomical classification of renal pelvicalyceal system based on three-dimensional (3D) virtual reconstruction models for endourological surgery.

Methods: We retrospectively collected data on 225 patients (320 kidneys) in total between Apr. 2017 and Dec. 2020, spatial anatomical structure of renal pelvis and calyces were modeled and corresponding morphological parameters were measured after $3 \mathrm{D}$ virtual reconstruction of computed tomography urography (CTU). The modified Takazawa renal pelvicalyceal anatomical classification was advanced based on the renal pelvicalyceal morphological parameters [bifurcated branches of renal pelvis, cross sectional area of renal pelvis and ureteropelvic junction (UPJ), infundibuloureteral angle (IUA), lower pole infundibular calyceal length (IL)] by 3D virtual reconstruction models, and comparison of renal pelvicalyceal system morphological parameters were performed to evaluate the differences in various classification types of renal pelvis and calyces.

Results: Anatomical structure of renal pelvis and calyces were divided into two main types (Type A and Type B) according to renal pelvic branch patterns. A single pelvis without bifurcated branch was regarded as Type A (62\%) and subclassified into three subtypes: Type A1 (22\%), Type A2 (27\%) and Type A3 (13\%), the slimline pelvis was classified as Type A1, the typical pelvis as Type A2 and the broad pelvis as Type A3. A divided pelvis with bifurcated branches was seen as Type B (38\%) and subclassified into two subtypes: Type B1 (15\%) with the wide and flat lower calyx branch, Type B2 (23\%) with the narrow and steep lower calyx branch.

Conclusions: Previous studies have reported that the visualization and classification of renal pelvicalyceal anatomical structure by endocast, autopsy, ultrasonography and excretory urography, the modified Takazawa classification system based on 3D virtual reconstruction models enables to standardized different anatomical morphology of renal pelvicalyceal system and provide intuitive and concise information on anatomy, thus leading to the improvement in treatment modality.

Keywords: Renal pelvicalyceal system; renal anatomy classification; three-dimensional virtual reconstruction; urolithiasis; endourology

\footnotetext{
$\wedge$ ORCID: 0000-0003-2699-8377.
} 
Submitted Apr 13, 2021. Accepted for publication Jun 02, 2021.

doi: 10.21037/tau-21-309

View this article at: https://dx.doi.org/10.21037/tau-21-309

\section{Introduction}

In recent years, advances in endourology, interventional radiology and percutaneous nephrolithotomy (PCNL) have rekindled interest in the anatomy of renal pelvicalyceal system $(1,2)$, as a comprehensive understanding of such anatomy is essential to achieve reliable endourologic surgery using for the treatment of urolithiasis, upper urinary tract urothelial tumors and chronic unilateral hematuria (3-6).

When we analyze the structure of kidney, we observe that the pelvis and calyces are the most varied in number, position and shape. Renal pelvicalyceal anatomy may play a role in the selecting the best endourologic treatment for a particular patient. It is necessary to fully understand the three-dimensional (3D) renal pelvicalyceal anatomical structure, in order to interpret the preoperative images accurately and complete the operation effectively and safely.

Brödel reported the anatomy of the renal pelvis and vessels in 1901 and he divided the renal pelvis into two types: the true pelvis and the divided pelvis (7), some following renal pelvicalyceal classifications have been proposed progressively. However, we think that several important details of pelvicalyceal anatomy still have not been discussed, these classifications do not assist clinical endourologic applications and the morphologic types they grouped deserve to be described thoroughly. To visualize the renal pelvicalyceal system in three dimensions and help urologists to image the characteristic of the renal calyces for a better chose of therapeutic option, we systematically studied the $3 \mathrm{D}$ virtual reconstruction models of the renal pelvis and calyces and put forward modified Takazawa kidney collecting system morphologic classification of clinical value to endourologists. We present the following article in accordance with the STROBE reporting checklist (available at https://dx.doi.org/10.21037/tau-21-309).

\section{Methods}

\section{Study object}

We retrospectively collected data on a total of 320 renal pelvicalyceal systems from computed tomography urography (CTU) of 225 patients, who underwent urological imaging examinations due to malignant or benign disease of urinary system at our hospital during the period from Apr. 2017 to Dec. 2020. 3D virtual reconstruction models of hydronephrosis, urinary tract malformation, or poor image quality were excluded. All procedures performed in this study were in accordance with the Declaration of Helsinki (as revised in 2013) and approved by the Ethics Committee of Peking University First Hospital (approval number: 2020-346). Because of the retrospective nature of the research, the requirement for informed consent was waived.

\section{CT scan and 3D virtual reconstruction models}

Enhanced CT scans were completed in all patients using a 64-slice CT system with $0.625 \mathrm{~mm}$ slice thickness (Light-Speed VCT, GE Healthcare, USA), the original CT data for the following analysis were set to the image quality and stored in DICOM (Digital Imaging and Communications in Medicine) format. Different phase CT data were segmented and reconstructed to create 3D digital images through Medical Imaging Three Divisional Visualization System (Yorktal, Inc., Shenzhen, Guangdong, China). The threshold value method was used to extract a targeted region. 3D virtual reconstruction models of the renal pelvicalyceal system were obtained based on secretive phase data, while other retroperitoneal anatomical structures including renal parenchyma, renal artery, vein, abdominal aorta and inferior vena cava were reconstructed simultaneously using the region-growing method based on arterial and parenchymal phase data (8).

\section{Data collection and statistics analysis}

We measured characteristics of the renal pelvicalyceal system, including side of kidney collecting system, number of renal calyces, cross-sectional area of renal pelvis, angle between the central axis of renal pelvis and upper ureter, infundibulopelvic angle (IPA), infundibuloureteral angle (IUA) and infundibular calyceal length (IL). We then put forward the modified Takazawa anatomical classification based on the renal pelvicalyceal morphological parameters mentioned above and compared the difference of the renal pelvicalyceal types. The angle was measured as described by Elbahnasy et al. (9). And in our study, the cut-off value of 
Table 1 Morphological parameters of all modified Takazawa renal pelvicalyceal classification types

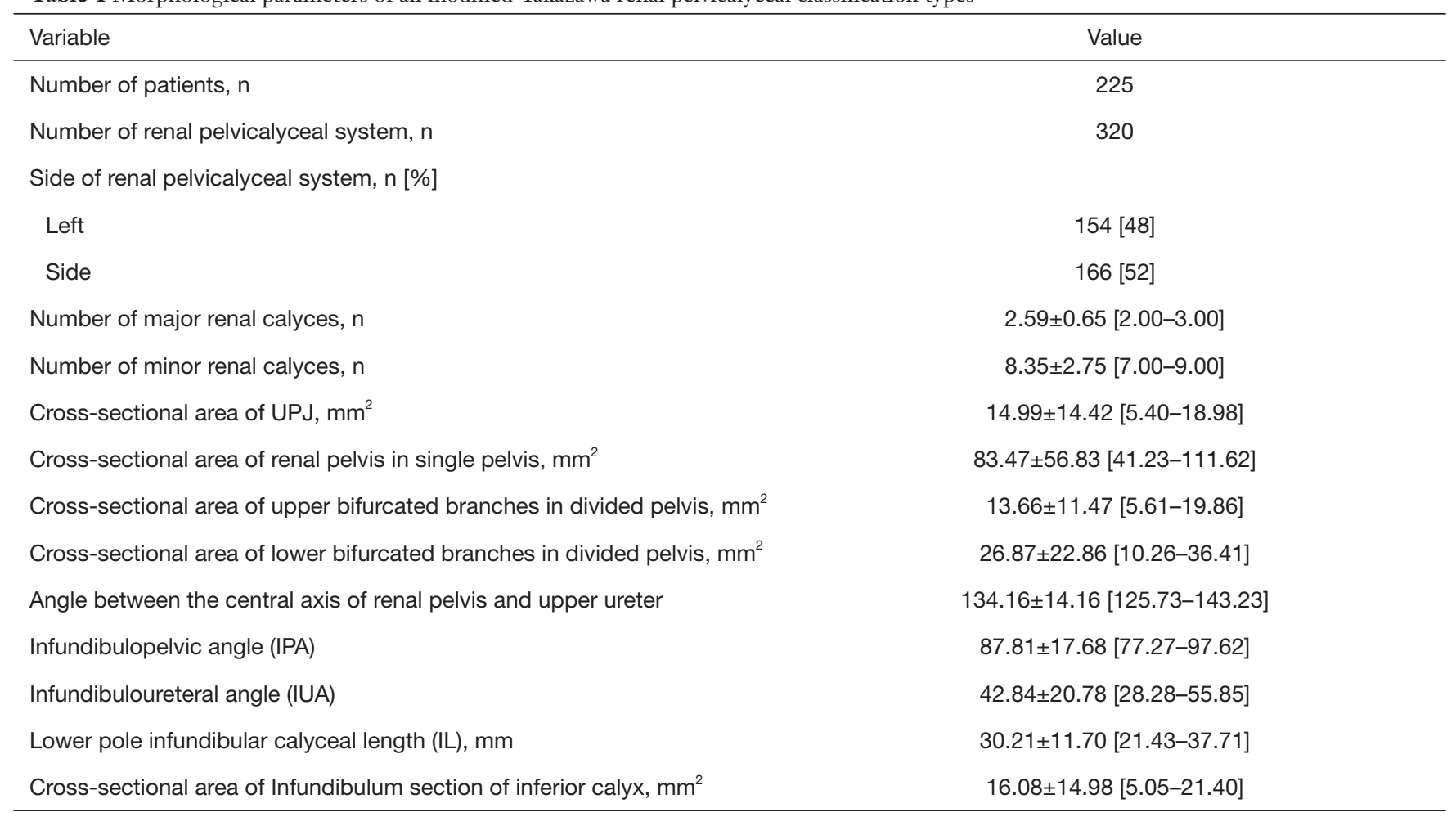

Data are presented in terms of mean \pm standard deviation [inter quartile range] or the numbers of individuals. UPJ, ureteropelvic junction.

classification and sub-classification was based on previous literature and our data distribution.

Statistical analyses were conducted by means of SPSS version 22.0 software (SPSS, Inc., IBM Corp., Somers, NY). Mean and median were calculated for descriptive purposes. Although we are consistent in measurement technique, people may argue about the end of each measurement. The measurement repeated by a second experienced individual researcher could minimize intra-observer variability.

\section{Results}

Of the 320 renal pelvicalyceal system from 225 patients were included as study subjects and analyzed, according to inclusion and exclusion criteria (Table 1). Anatomical structure of renal pelvis and calyces were divided into two major types (Type A and Type B) according to the renal pelvic branch patterns (Figure 1). Type A (198/320, 62\%) was present a single pelvis without bifurcated branch and subclassified into three subtypes according to the crosssectional area of renal pelvis (Figure $2 A$ ): Type A1 (70/320, $22 \%)$, Type A2 (86/320, 27\%) and Type A3 (42/320, 13\%).
If the cross-sectional area ratio of the pelvis to the UPJ was less than 4 times, the pelvis was considered to be type A1, which was a slimline pelvis morphology. The type A2 formed a typical funnel shaped pelvis and the cross-sectional area of the pelvis was about 4-16 times larger than that of the UPJ, and this subtype was the most frequently observed standard morphology. The type A3 subtype was a broad pelvis morphology which formed a large box shaped pelvis with the cross-sectional area ratio of the pelvis to the UPJ greater than 16 times.

A divided pelvis with bifurcated branches was seen as Type B (122/320, 38\%) and subclassified into two subtypes: Type B1 $(48 / 320,15 \%)$ with the wide and flat lower calyx infundibulum, and Type B2 (74/320, 23\%) with the narrow and steep lower calyx infundibulum (Figure 2B). If two or three of the following three conditions are met: renal lower bifurcated branches of divided pelvis-to-UPJ cross-sectional area ratio $>4$, IUA $>30^{\circ}, \mathrm{IL}<3 \mathrm{~cm}$, the pelvis was considered to be type $\mathrm{B} 1$ which is relatively easy for lithotripsy. If none or one of the following three conditions mentioned above are met, the pelvis was considered to be type B2 which is relatively hard for lithotripsy. Table 2 showed a summary 

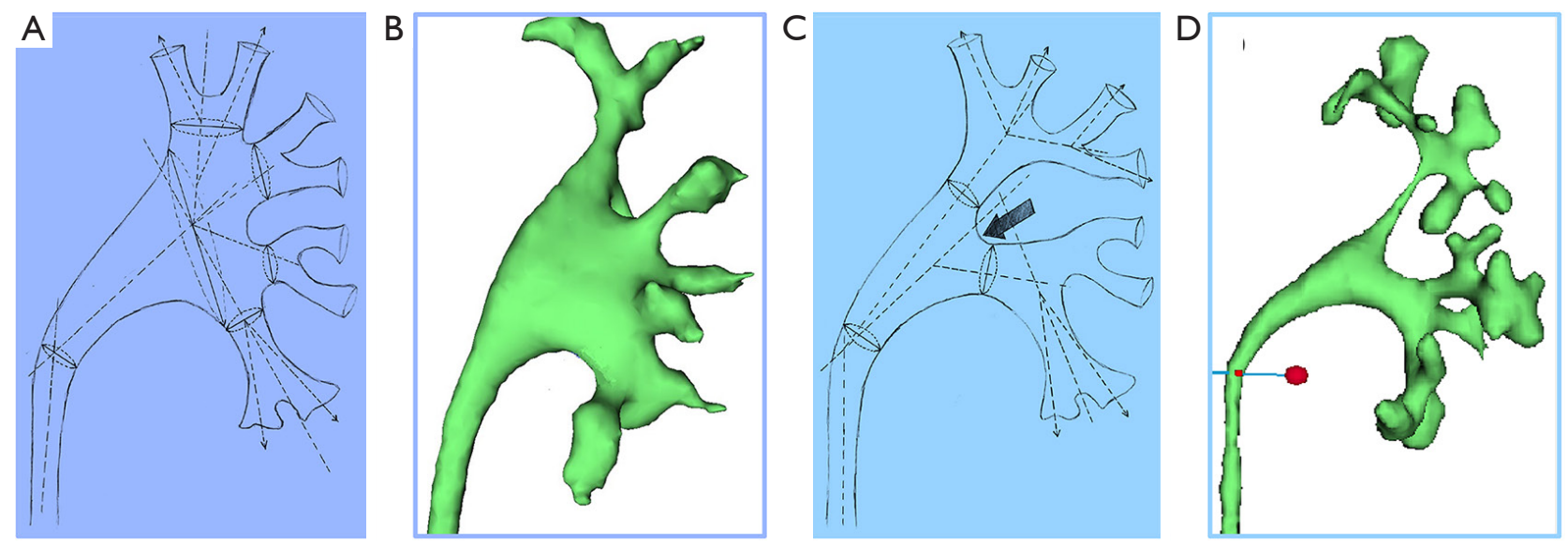

Figure 1 Model diagram and three-dimensional virtual reconstruction models of two modified Takazawa renal pelvicalyceal anatomical classification types. (A) Model diagram depicting Type A; (B) three-dimensional virtual reconstruction model of Type A; (C) model diagram depicting Type B; (D) three-dimensional virtual reconstruction model of Type B.
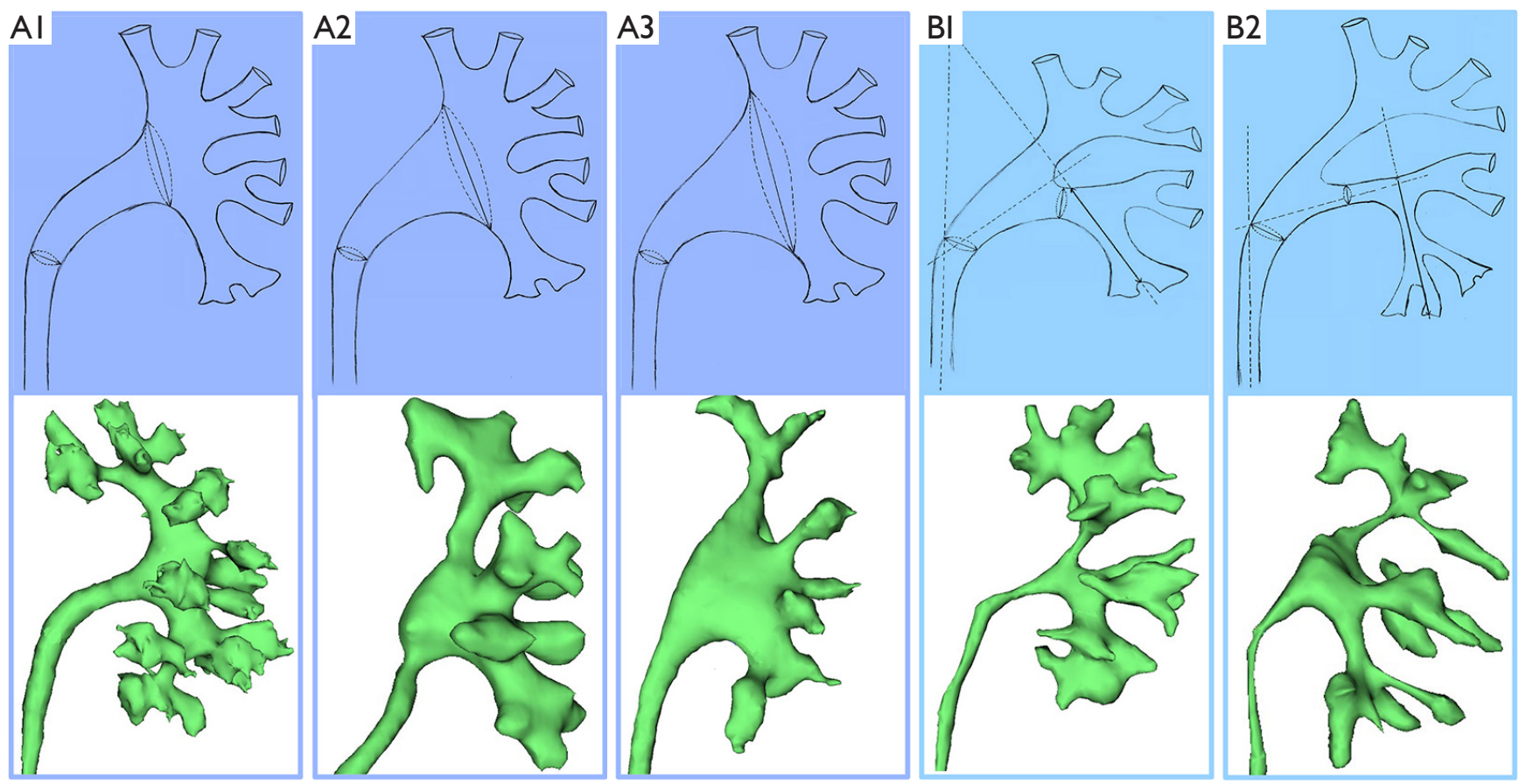

Figure 2 Model diagrams and three-dimensional virtual reconstruction models of five modified Takazawa renal pelvicalyceal anatomical classification subtypes. (A1) Type A1-renal pelvis-to-ureteropelvic junction (UPJ) cross-sectional area ratio $\leq 4$. (A2) Type A2-renal pelvis-to-UPJ cross-sectional area ratio $=4-16$. (A3) Type A3-renal pelvis-to-UPJ cross-sectional area ratio $\geq 16$. (B1) Type B1-two or three of the following three conditions are met [renal lower bifurcated branches of divided pelvis-to-UPJ cross-sectional area ratio $>4$, infundibuloureteral angle (IUA) $>30^{\circ}$, lower pole infundibular calyceal length (IL) $\left.<3 \mathrm{~cm}\right]$. (B2) Type B2-none or one of the following three conditions are met [renal lower bifurcated branches of divided pelvis-to-UPJ cross-sectional area ratio $>4$, IUA $>30^{\circ}$, lower pole infundibular calyceal length (IL) $<3 \mathrm{~cm}]$. 
Table 2 Summary of the modified Takazawa anatomical classification of renal pelvicalyceal system based on three-dimensional virtual reconstruction models

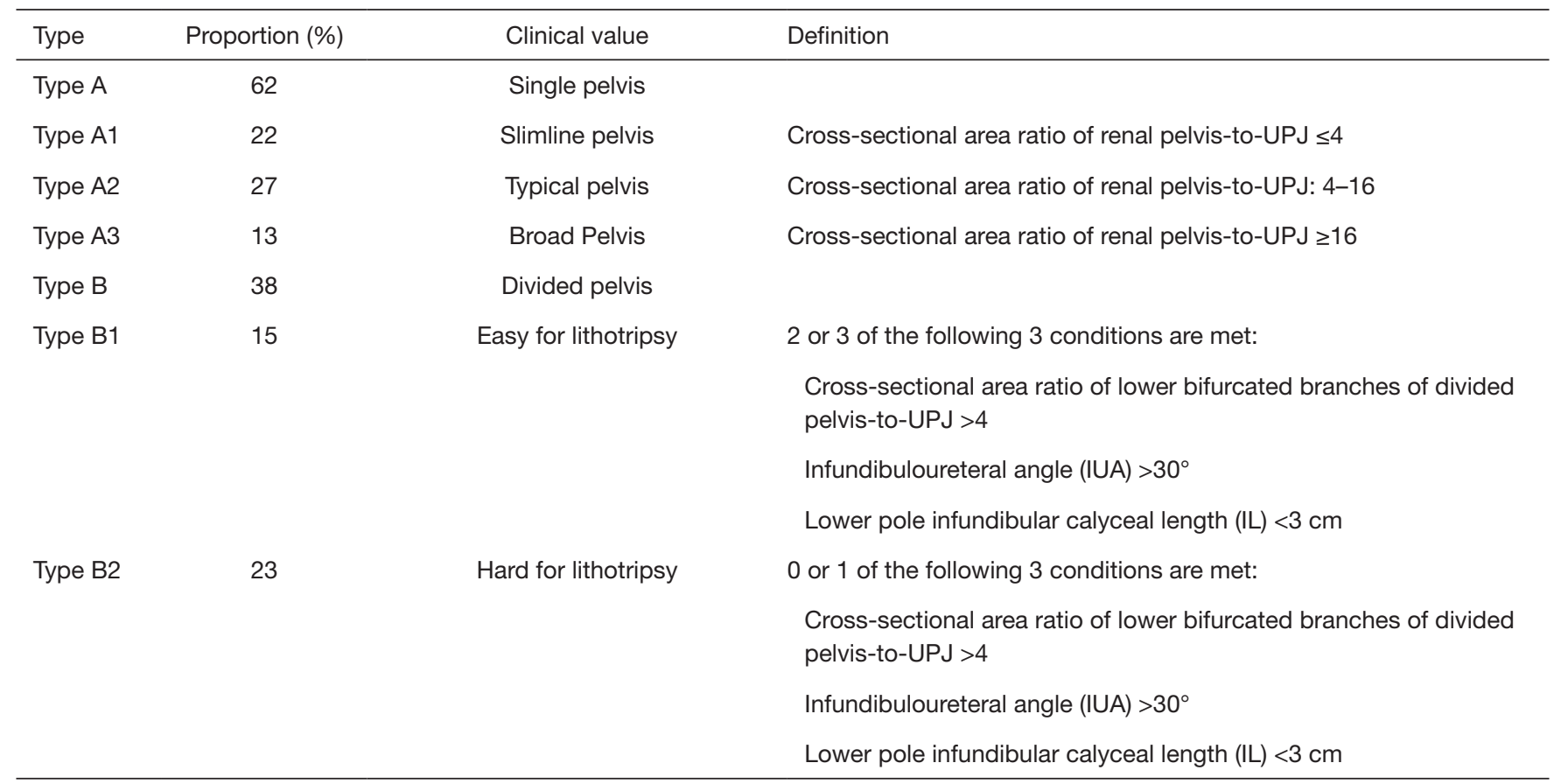

UPJ, ureteropelvic junction.

of the modified classification system, and Table 3 showed comparing morphological parameters of all modified renal pelvicalyceal classification types.

\section{Discussion}

The pelvicalyceal system consists of minor calyces, major calyces and the renal pelvis. Calyx is the cavity of the kidney, through which urine passes. The minor calyx is $7-13$ in number and cup-shaped, surrounding the apex of renal pyramid (10). The neck of the minor calyx is a slimline structure, which is called infundibulum. Two or three minor calyces converge to form a major calyx, which enters renal pelvis with a certain angle (11). The pelvis is the funnel, like the upper end of a dilated ureter. The renal pelvis, the major calyces, the infundibula and the minor calyces are collectively called intrarenal pelvicalyceal system.

One of the most easily neglected aspects is the anatomy of renal pelvicalyceal system, during endourological removal of renal stone. Although many studies have reached some conclusions on the related factors, the gross anatomic variation of the renal pelvicalyceal system might be as numerous as fingerprint of an individual $(12,13)$. The symmetry of the renal pelvicalyceal system of a single individual on both sides is only about $37 \%$ (14). Not only is the position and number of different parts of the renal pelvicalyceal system different among individuals, but the parts could either be numerous or absent as well. The applied anatomy of the renal pelvicalyceal system, especially for the lower pole and inferior calyces, is still lack of large sample size analysis.

Surgeons, especially the junior with less experience in endourologic treatment, may disorientated in the renal pelvicalyceal system because they are not familiar with the anatomical characteristics of renal pelvis and calyces, which may affect the surgical efficacy, cause residual stones and even iatrogenic injury. For senior surgeons, although they may be familiar with the renal pelvicalyceal system, however due to the lack of scientific data support, these empirical understanding is often difficult to be promoted to the theoretical level. A comprehensive understanding of renal pelvicalyceal anatomy is essential for the interpretation of preoperative imaging examination and reliable endourological surgery. In order to help urologists to image the exact characteristics of the calyces and visualize the renal pelvicalyceal system in 3 dimensions, we conducted this systematical study of renal pelvicalyceal anatomy and detailed the modified Takazawa classification of that from 
Table 3 Morphological parameters of various modified Takazawa renal pelvicalyceal classification types

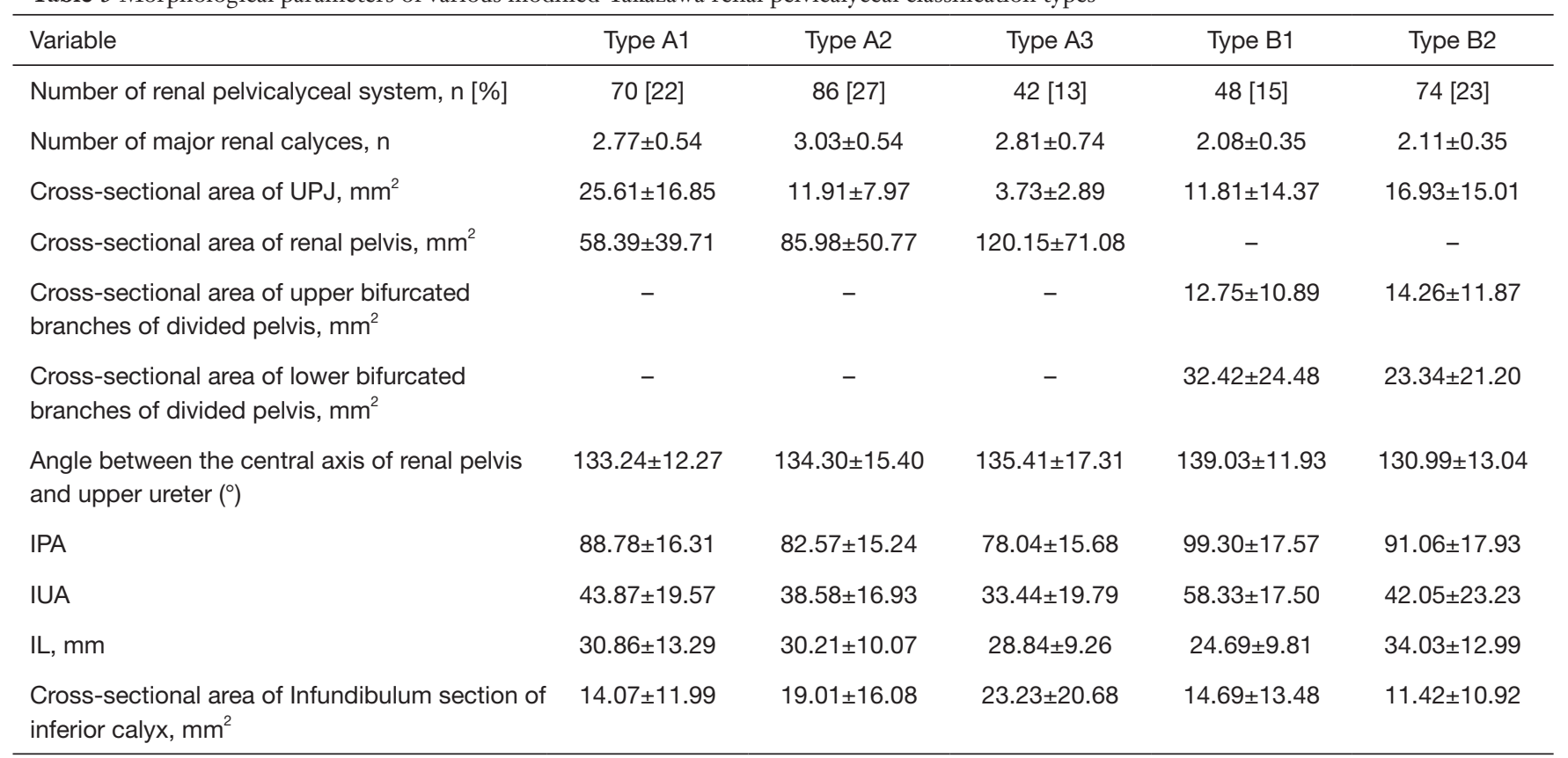

Data are presented in terms of mean \pm standard deviation or the numbers of individuals. UPJ, ureteropelvic junction; IPA, infundibulopelvic angle; IUA, infundibuloureteral angle; IL, lower pole infundibular calyceal length.

3D virtual reconstruction models.

The kidney collecting system can be divided into many types according to the position and shape of renal pelvis, the pattern and length of drainage of calyces (Table 4). Bruce Sir et al. (15) observed that the location of renal pelvis as extrarenal, borderline or intrarenal. Graves (16) classified the pelvicalyceal patterns in different types according to the shape of the renal pelvis along with calyceal protrusion. Sampaio and Mandarim-de-Lacerda (14), based on polyester endocasts, provided the classification of the pelvicalyceal patterns in a variety of dimensions. They divided the pelvicalyceal system into four types according to the drainage of the mid (hilar) zone and of the polar regions. And Ningthoujam et al. (12) proposed another classification system, including bicalyceal, tricalyceal, and multicalyceal types. As far as the limitations of previous classifications of renal pelvicalyceal system are concerned, most classifications were based on endocasts which could change their shape on hardening, or based on two-dimensional images which may be not help for spatial anatomical visualization. The reason why the most of classifications are not sufficiently widespread is probably because it is difficult to understand the corresponding anatomical parameters of renal pelvis and calyces intuitively and precisely, and because it is limited in clinical application, especially in endourological surgery.

In recent years, Japanese scholars Ryoji Takazawa et al. (17) proposed a nomenclature for renal calices, and simplified morphological classifications of the renal pelvis suitable for endourological surgery. The classification proposed by Ryoji Takazawa et al. makes up for the limitations of previous studies on renal pelvis and calyces, they classified minor calyx into five levels : top, upper, middle, lower, and bottom, which make it possible to share common intrarenal information. They also divided the renal pelvis into two major types based on Brödel's research: single pelvis and divided pelvis. Single pelvis was subdivided into three types based on the width of the single pelvis. This classification system could be useful for the planning endoscopic treatment strategies for renal stones. By means of analyzing, analogizing and summarizing the data of our center, we once again recognized the correctness of Takazawa's and Brödel's findings and proposed a modified Takazawa anatomical classification of renal pelvicalyceal system based on 3D virtualization technology. Our proposed two major types were consistent with their findings and we found the ratio of the two main types is similar to that of Japanese scholars. What is different and innovative, we incorporated the parameters of $3 \mathrm{D}$ virtual reconstruction 
Table 4 Summary of renal pelvicalyceal classifications

\begin{tabular}{|c|c|}
\hline Studies & Classifications \\
\hline \multirow[t]{3}{*}{ Bruce Sir 1967, (15) } & Intrarenal \\
\hline & Extrarenal \\
\hline & Borderline \\
\hline \multirow[t]{4}{*}{ Grave 1954, (16) } & Classic Y, Type A \\
\hline & Inverted T, Type B \\
\hline & Balloon, Type C \\
\hline & Bagpipes, Type D \\
\hline \multirow[t]{4}{*}{ Sampaio 1988, (14) } & Type A-I \\
\hline & Type A-II \\
\hline & Type B-I \\
\hline & Type B-II \\
\hline \multirow[t]{3}{*}{ Ningthoujam 2005, (12) } & Bicaliceal (Y-shaped) \\
\hline & Tricaliceal (Triangular) \\
\hline & Multicaliceal (Radiate) \\
\hline \multirow[t]{4}{*}{ Takazawa 2018, (17) } & Type la \\
\hline & Type lb \\
\hline & Type Ic \\
\hline & Type II \\
\hline \multirow[t]{5}{*}{ Present study } & Type A1 \\
\hline & Type A2 \\
\hline & Type A3 \\
\hline & Type B1 \\
\hline & Type B2 \\
\hline
\end{tabular}

models as the basis for classification, meanwhile we described, analyzed and summarized the anatomy of lower pole calyx and infundibulum. We subclassified type B into two subtypes: Type B1 (15\%) with the wide and flat lower calyx branch, Type B2 (23\%) with the narrow and steep lower calyx branch which further improves the value of clinical application especially for lower pole calyceal calculi.

We believe that subclassification of type $B$ will also be practical for endourological treatment strategies for lower pole calyx stones, since the stone treatment in the lower pole calyx is less successful and more difficult compared with other parts of the kidney (18). Research on the relationship between renal pelvicalyceal system anatomical characteristics and urolithiasis began with the pioneering study of Aragao and Sampaio (19). Following studies have showed some lower pole pelvicalyceal anatomical features, such as infundibular calyceal width (IW), IL and IUA, which are important for stone clearance after retrograde intrarenal surgery (RIRS) and extracorporeal shockwave lithotripsy (ESWL). According to Elbahnasy (9), the following factors are believed to be beneficial to the removal of lower pole stones: IW $>5 \mathrm{~mm}$ and IL $\leq 3 \mathrm{~cm}$. On the contrary, IW $\leq 5 \mathrm{~mm}$ and $\mathrm{IL}>3 \mathrm{~cm}$ are considered as unfavorable factors. Geavlete et al. concluded that the IUA influences the performances of flexible ureteroscopy. They found that the larger the angle, the higher the success rate of lithotripsy in the treatment of lower calyces. When the angle is less than 30 degrees, it is usually difficult to treat the lower calyceal calculi with flexible ureteroscopy (20). Type B1 with flat IUA, large IW, and short IL is very likely to have complete removal of stone fragments, which is an appropriate case for RIRS and ESWL. On the other hand, the anatomic condition of type B2 patients is unfavorable, especially for narrow, long and steep infundibular, residual stones after ESWL are almost inevitable. Under these circumstances, alternative therapies such as ureteroscopy lithotripsy or PCNL are preferable, as these procedures are relatively insensitive to the negative anatomical factors. As far as we know, there has not been the research on the topic, and we have reported subclassification of lower pole infundibulum initially based on $3 \mathrm{D}$ virtual reconstruction models, which is of clinical value for clearance of lower pole renal calculi.

In accordance with the study of Takazawa, under the circumstance of Type A1, which is narrow pelvis, PCNL seems hard than typical or broad pelvis due to the restricted space. While ureteroscopy is performed in type A1, surgeons should pay more attention to distinguish renal pelvis from the ureter in order to avoid damage of renal pelvicalyceal system. Type A3, the broad pelvis, seems easier for PCNL to make relatively larger size tract. On the other hand, this type is easily confused with hydronephrosis and when ureteroscopy lithotripsy performed, stone dust or fragments may deposit down to the bottom.

Fine knowledge of the anatomy remains the cornerstone of surgery (2). The main limitation of the previous studies was the two-dimensional features by anatomical measurements through urography (21). In our study, CTU makes it possible to perform measurements in $3 \mathrm{D}$ virtual reconstruction models, which has proved to be a useful method for analyzing intrarenal and extrarenal anatomical characteristics $(22,23)$. In previous studies, liquid rubber 
was injected into the renal pyelocaliceal system to produce endocast reprints. The technique can clarify the intrarenal relationship between the injected cavities but neglected the extrarenal relationship with the rest of the body. Due to the shrinkage of rubber after hardening or expansion at higher pressure while injecting, it is also easy to produce errors $(14,24,25)$. In our study, 3D virtual reconstruction models can make up for the above two defects of endocast, and could enable a clear understanding of the pelvicalyceal anatomy.

However, our study had several limitations. First, our study is retrospective and limited to the 320 images of kidneys after approximately $12 \%$ of kidneys were excluded due to anatomical distortion (hydronephrosis or large renal cysts) or insufficient image quality. Similar to the research of Japanese scholars, our research objects are all East Asian races, thus prospective research covering different races needs to be further carried out. Second, by referring to the previous literatures on the anatomy of kidney collective system, we analyzed, analogized and summarized the data of our center, and then put forward the modified Takazawa anatomical classification. However, describing the classification based on the location of calyces and the shape of pelvis, it is hardly novel. By the means of $3 \mathrm{D}$ virtual reconstruction models, we made efforts to involve anatomical information of the lower pole infundibulum and to provide spatial anatomical parameters such as area of renal pelvis and UPJ, so that urologists can intuitively understand the anatomy and make preoperative plan for endourological lithotripsy. Third, the clinical utility of the modified classification system needs further research. Although many studies have compared different treatment options for kidney stones, most studies have not studied the renal pelvicalyceal anatomy that would predict the success of these treatments. In the next step, we plan to validate the system in a multicenter study and explore the impact of each morphological classification on endourological treatment efficacy.

\section{Conclusions}

Previous studies have reported that the visualization and classification of renal pelvicalyceal anatomical structure by corrosion cast, autopsy, ultrasonography and excretory urography, the modified Takazawa anatomical classification of renal pelvicalyceal system based on $3 \mathrm{D}$ virtual reconstruction models enables to standardized different anatomical morphology of renal pelvicalyceal system and provide intuitive and concise information of lower pole infundibulum anatomy, therefore leading to the improvement of endourological lithotripsy.

\section{Acknowledgments}

The authors thank the entire staff of the Department of Urology, Peking University First Hospital.

Funding: None.

\section{Footnote}

Reporting Checklist: The authors have completed the STROBE reporting checklist. Available at https://dx.doi. org/10.21037/tau-21-309

Data Sharing Statement: Available at https://dx.doi. org/10.21037/tau-21-309

Conflict of Interest: All authors have completed the ICMJE uniform disclosure form (available at https://dx.doi. org/10.21037/tau-21-309). Dr. XL serves as an unpaid editorial board member of Translational Andrology and Urology currently. The authors have no conflicts of interest to declare.

Ethical Statement: The authors are accountable for all aspects of the work in ensuring that questions related to the accuracy or integrity of any part of the work are appropriately investigated and resolved. All procedures performed in this study were in accordance with the Declaration of Helsinki (as revised in 2013) and approved by the Ethics Committee of Peking University First Hospital (approval number: 2020-346). Because of the retrospective nature of the research, the requirement for informed consent was waived.

Open Access Statement: This is an Open Access article distributed in accordance with the Creative Commons Attribution-NonCommercial-NoDerivs 4.0 International License (CC BY-NC-ND 4.0), which permits the noncommercial replication and distribution of the article with the strict proviso that no changes or edits are made and the original work is properly cited (including links to both the formal publication through the relevant DOI and the license). See: https://creativecommons.org/licenses/by-nc-nd/4.0/. 


\section{References}

1. Kaye KW. Renal anatomy for endourologic stone removal. J Urol 1983;130:647-8.

2. Kaye KW, Reinke DB. Detailed caliceal anatomy for endourology. J Urol 1984;132:1085-8.

3. Giusti G, Proietti S, Peschechera R, et al. Sky is no limit for ureteroscopy: extending the indications and special circumstances. World J Urol 2015;33:257-73.

4. Rouprêt M, Babjuk M, Compérat E, et al. European guidelines on upper tract urothelial carcinomas: 2013 update. Eur Urol 2013;63:1059-71.

5. Tanimoto R, Kumon H, Bagley DH. Development of Endoscopic Diagnosis and Treatment for Chronic Unilateral Hematuria: 35 Years Experience. J Endourol 2017;31:S76-S80.

6. Takazawa R, Kitayama S, Tsujii T. Digital ureteroscopic visualization of lesions responsible for chronic unilateral hematuria, so-called idiopathic renal bleeding. Int J Urol 2014;21:227-8.

7. Brödel M. The intrinsic blood-vessels of the kidney and their significance in nephrotomy. 1901. Available online: https://collections.nlm.nih.gov/catalog/nlm:nlmuid101648447-bk

8. Teber D, Guven S, Simpfendörfer T, et al. Augmented reality: a new tool to improve surgical accuracy during laparoscopic partial nephrectomy? Preliminary in vitro and in vivo results. Eur Urol 2009;56:332-8.

9. Elbahnasy AM, Shalhav AL, Hoenig DM, et al. Lower caliceal stone clearance after shock wave lithotripsy or ureteroscopy: the impact of lower pole radiographic anatomy. J Urol 1998;159:676-82.

10. Ryan S, McNicholas M, Eustace SJ. Anatomy for diagnostic imaging e-book. Elsevier Health Sciences, 2011.

11. Hanif MS, Toori MH, Sheikh M. Detailed calyceal anatomy for endourology. Pakistan J Med Res 2004;43:184-7.

12. Ningthoujam DD, Chongtham RD, Sinam SS. Pelvicalyceal pattern in foetal and adult human kidneys. Journal of the Anatomical Society of India 2005;54:1-11.

13. Kaye KW, Goldberg ME. Applied anatomy of the kidney and ureter. Urol Clin North Am 1982;9:3-13.

14. Sampaio FJB, Mandarim-de-Lacerda CA. Anatomic classification of the kidney collecting system for endourologic procedures. J Endourol 1988;2:247-51.

15. Bruce Sir J, Warmsley R, Ross J. The abdominal cavity in manual of surgical anatomy. Edinburgh: $\mathrm{E}$ and $\mathrm{S}$ Livingstone Ltd, 1967.

16. Graves FT. The anatomy of the intrarenal arteries and its application to segmental resection of the kidney. Br J Surg 1954:42:132-9.

17. Takazawa R, Kitayama S, Uchida Y, et al. Proposal for a Simple Anatomical Classification of the Pelvicaliceal System for Endoscopic Surgery. J Endourol 2018;32:753-8.

18. Karim SS, Hanna L, Geraghty R, et al. Role of pelvicalyceal anatomy in the outcomes of retrograde intrarenal surgery (RIRS) for lower pole stones: outcomes with a systematic review of literature. Urolithiasis 2020;48:263-70.

19. Sampaio FJ, Aragao AH. Inferior pole collecting system anatomy: its probable role in extracorporeal shock wave lithotripsy. J Urol 1992;147:322-4.

20. Geavlete P, Multescu R, Geavlete B. Influence of pyelocaliceal anatomy on the success of flexible ureteroscopic approach. J Endourol 2008;22:2235-9.

21. Binbay M, Akman T, Ozgor F, et al. Does pelvicaliceal system anatomy affect success of percutaneous nephrolithotomy? Urology 2011;78:733-7.

22. El-Assmy A, Abo-Elghar ME, El-Nahas AR, et al. Anatomic predictors of formation of lower caliceal calculi: is it the time for three-dimensional computed tomography urography? J Endourol 2008;22:2175-9.

23. Miller J, Durack JC, Sorensen MD, et al. Renal calyceal anatomy characterization with 3-dimensional in vivo computerized tomography imaging. J Urol 2013;189:562-7.

24. Sampaio FJ, Mandarim-De-Lacerda CA, De Aragao AH. The collector system of the kidney. Applied anatomy based on the analysis of 3-dimensional casts. J Urol (Paris) 1987;93:183-5.

25. Sampaio FJ. Renal collecting system anatomy: its possible role in the effectiveness of renal stone treatment. Curr Opin Urol 2001;11:359-66.

Cite this article as: Zhu W, Zheng $M$, Xiong S, Han G, Meng C, Li Z, Zhang L, Xiong G, Guan H, Huang Y, Zhu H, Li X, Wang G, Zhou L. Modified Takazawa anatomical classification of renal pelvicalyceal system based on threedimensional virtual reconstruction models. Transl Androl Urol 2021;10(7):2944-2952. doi: 10.21037/tau-21-309 